\title{
Research on Optimized Torque-Distribution Control Method for Front/Rear Axle Electric Wheel Loader
}

\author{
Zhiyu Yang, Jixin Wang, Guangzong Gao, and Xiangyun Shi \\ School of Mechanical Science and Engineering, Jilin University, Changchun 130025, China \\ Correspondence should be addressed to Jixin Wang; jxwang@jlu.edu.cn
}

Received 7 April 2017; Revised 18 July 2017; Accepted 27 July 2017; Published 10 September 2017

Academic Editor: Nunzio Salerno

Copyright (C) 2017 Zhiyu Yang et al. This is an open access article distributed under the Creative Commons Attribution License, which permits unrestricted use, distribution, and reproduction in any medium, provided the original work is properly cited.

Optimized torque-distribution control method (OTCM) is a critical technology for front/rear axle electric wheel loader (FREWL) to improve the operation performance and energy efficiency. In the paper, a longitudinal dynamics model of FREWL is created. Based on the model, the objective functions are that the weighted sum of variance and mean of tire workload is minimal and the total motor efficiency is maximal. Four nonlinear constraint optimization algorithms, quasi-newton Lagrangian multiplier method, sequential quadratic programming, adaptive genetic algorithms, and particle swarm optimization with random weighting and natural selection, which have fast convergent rate and quick calculating speed, are used as solving solutions for objective function. The simulation results show that compared to no-control FREWL, controlled FREWL utilizes the adhesion ability better and slips less. It is obvious that controlled FREWL gains better operation performance and higher energy efficiency. The energy efficiency of FREWL in equipment transferring condition is increased by $13-29 \%$. In addition, this paper discussed the applicability of OTCM and analyzed the reason for different simulation results of four algorithms.

\section{Introduction}

Hybrid wheel loader has raised much attention due to its green technology. It is considered to be the trend of future in the loader field [1-3]. Here are some released hybrid loader prototypes of several manufacturers, as shown in Table 1.

However, the energy-saving method of these above loaders is energy management strategy [4-6]. Besides, its dynamic performance, passing performance, and operation efficiency have no obvious difference with conventional diesel driven wheel loader. Hence, optimized torque-distribution control method (OTCM) of front/rear drive axle or four wheels is essential to improve operation efficiency, providing a new energy-saving strategy $[7,8]$. Considering the cost and control technology, the configuration of front/rear axle independent drive is more possible to realize mass production than four-wheel drive, like mass-produced electric vehicles Tesla Model S [9] and BYD QIN [10].

There are many technologies demanding prompt solution about OTCM for FREWL. Enlightened by the relative research in on-road vehicle field, tire energy dissipation [11], total motor efficiency [12], and motor power loss [13] are used as energy efficiency optimization objective. Tire workload reflects the utilization of road adhesion ability [14]. Through the control of tire workload, the operation performance of FREWL is obviously improved.

In this paper, the proposed OTCM for FREWL is to gain better operation performance and higher energy efficiency. In the primary stage of FREWL dynamics research, it is more urgent to study longitudinal dynamics than to study the lateral stability because FREWL is often operated in low speed. This paper assumes that FREWL only moves in the longitudinal direction. In Section 2, the dynamic model is created based on the configuration of FREWL. In Section 3, depending on the target to improve the operation performance and energy efficiency, objective functions are that the weighted sum of variance and mean of tire workload is minimal and the total motor efficiency is maximal. Then constraint conditions of the optimization control are listed. Four nonlinear optimization algorithms with constraints, quasinewton Lagrangian multiplier method (QNLM), sequential quadratic programming (SQP) $[14,15]$ adaptive genetic algorithms (AGA) [16, 17], and particle swarm optimization with random weighting and natural selection (PSO-RN) $[18,19]$, 
TABLE 1: Outline of several prototypes.

\begin{tabular}{lcccc}
\hline Manufacturer & Powertrain configuration & Energy storage devices & Energy saving & Ref \\
\hline Hitachi & Series & Battery & $25 \%-30 \%$ & $25 \%$ \\
John Deere & Series & Battery & $420-23]$ \\
Joy Global & - & Flywheel & {$[24]$} \\
Volvo & - & Battery & - & {$[25]$} \\
XCMG & Parallel & Hydraulic accumulator & $54 \%$ & {$[8]$} \\
Liu Gong & Series-parallel & Supercapacitor & - & {$[26]$} \\
\hline
\end{tabular}

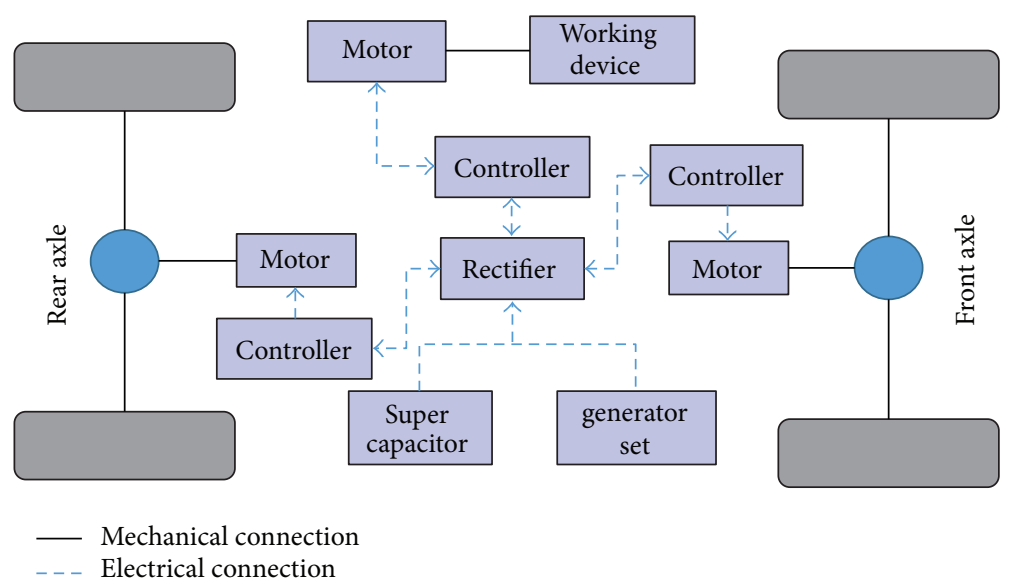

FIgURE 1: Transmission configuration of FREWL.

are introduced to solve the objective function. In Section 4, the effectiveness of OTCM is verified by simulation analysis. In Section 5, we discussed the applicability of OTCM and analyzed the reason for different simulation results of four algorithms.

\section{Dynamical Model of FREWL}

The distinctive transmission configuration of FREWL takes diesel generating set as main power source. Rectifier converts the alternating current generated by diesel generating set to a direct current which used to drive front motor, rear motor, and working motor. Supercapacitor is used as auxiliary source to effectively use braking energy and control diesel generating set in its high efficiency operating region. So diesel generating set can always operate in its high efficiency region. The transmission configuration of FREWL is shown in Figure 1.

A brief summary of the forces and torques in longitudinal dynamics is shown in Figure 2.

2.1. Wheel Vertical Load. Because FREWL operates in low speed, the influence of air resistance can be ignored. The wheel vertical load is, respectively, given by

$$
\begin{aligned}
F_{z f} & =\frac{m \dot{v}_{x} h-m g h \sin \alpha+m g l_{r} \cos \alpha+F_{z} l_{z}}{l_{f}+l_{r}} \\
F_{z r} & =\frac{-m \dot{v}_{x} h+m g h \sin \alpha+m g l_{f} \cos \alpha-F_{z} l_{c}}{l_{f}+l_{r}},
\end{aligned}
$$

where $l_{f}$ is the distance from FREWL gravity center to front axle, $l_{r}$ is the distance from FREWL gravity center to rear axle, $l_{c}$ is the distance from the front axle to the tooth tip of bucket, $l_{z}=l_{c}+l_{f}+l_{r}, h$ is the height of FREWL gravity center, $m$ is the mass, $\alpha$ is the gradient of the slope, $\dot{v}_{x}$ is the longitudinal acceleration, $F_{z f}$ and $F_{z r}$ are the vertical loads of front and rear wheels, $F_{z}$ is the vertical component of spading resistance on the tooth tip of bucket, and $F_{x}$ is the horizontal component of spading resistance on the tooth tip of bucket.

2.2. Tire Driving Torque and Wheel Longitudinal Force. The relationship between the longitudinal force and driving torque on each tire is given by

$$
I_{e} \dot{\omega}_{w i}=T_{i}-r_{\mathrm{eff}} F_{x i},
$$

where $I_{e}$ is the wheel rotational inertia, $r_{\text {eff }}$ is the tire rolling radius, $\dot{\omega}_{w i}$ is the wheel angular acceleration, $F_{x i}$ is the wheel longitudinal force, and $T_{i}$ is the tire driving torque. $i$ in subscript denotes the front or rear axle.

2.3. Motor Driving Torque. Suppose that longitudinal force and vertical load of the wheels in the same axle are equal; the relationship between the motor driving torque and tire driving torque is given by

$$
T_{m i}=\frac{2 T_{i}}{N}+I_{m} \dot{\omega}_{m i}
$$

where $T_{m i}$ is the motor driving torque, $I_{m}$ is the motor rotational inertia, $\dot{\omega}_{m i}$ is the motor angular acceleration, and $N$ is the reduction ratio from the motor to the wheels. 


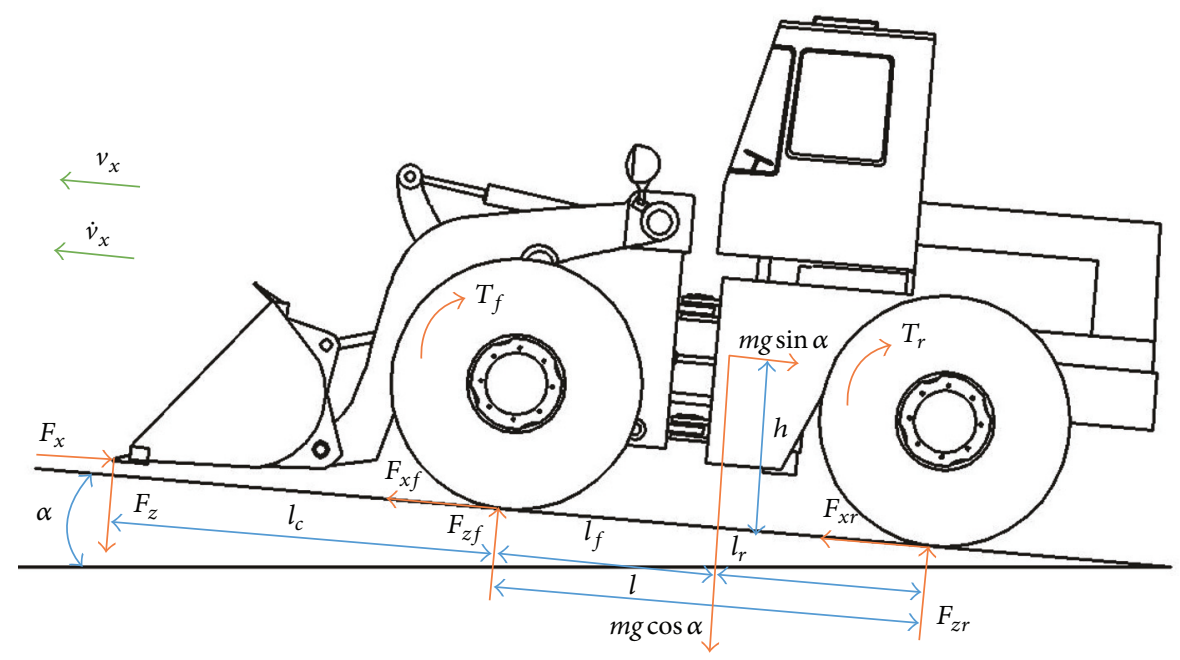

FIGURE 2: Illustration of FREWL forces and torques.

\section{Optimal Torque-Distribution Control Method}

OTCM consists of objective function, constraint conditions, and optimization algorithm.

\subsection{Objective Function}

3.1.1. Optimized Torque-Distribution Control Based on Tire Workload. The nonlinear optimization problem about enhancing operating performance can be formulated in this way. Because of the characteristic that the driving torque of both motors can be controlled online, the objective function is that the weighted sum of variance and mean of tire workload is minimal [5]. It can be defined as

$$
\begin{aligned}
\min \quad J & =\operatorname{var}\left(\gamma_{i}\right)+\varepsilon_{v} E\left(\gamma_{i}\right) \\
& =\frac{1}{4} \sum_{i=1}^{4}\left(\gamma_{i}-E\left(\gamma_{i}\right)\right)^{2}+\varepsilon_{v} E\left(\gamma_{i}\right) .
\end{aligned}
$$

From (2) and (3), tire workload $\gamma_{i}$ of each wheel is defined as

$$
\gamma_{i}=\frac{T_{m i}^{2} N^{2}}{4 r_{\mathrm{eff}}^{2} \mu_{i}^{2} F_{z i}^{2}},
$$

where $\mu_{i}$ is the tire-road friction coefficient of each wheel and $N$ is the reduction ratio. By the characteristic that the driving torque of both front motor and rear motor can be controlled online, the objective function can be set by the minimum of weighted sum of variance and mean of tire workload. Distribution coefficient $\kappa$ is defined as

$$
\kappa=\frac{T_{m f}}{T_{m f}+T_{m r}}=\frac{T_{m f}}{T_{m}},
$$

where $T_{m}$ is the sum of driving torque of the front motor and rear motor, $T_{m f}$ is the driving torque of the front motor, and
$T_{m r}$ is the driving torque of the rear motor. Tire workload of each wheel is defined as

$$
\begin{aligned}
& \gamma_{f l}=\gamma_{f r}=\frac{\kappa^{2} T_{m}^{2} N^{2}}{4 r_{\mathrm{eff}}^{2} \mu_{f}^{2} F_{z f}^{2}} \\
& \gamma_{r l}=\gamma_{r r}=\frac{(1-\kappa)^{2} T_{m}^{2} N^{2}}{4 r_{\mathrm{eff}}^{2} \mu_{r}^{2} F_{z r}{ }^{2}},
\end{aligned}
$$

where $\gamma_{f l}$ is the tire workload of left-front wheel, $\gamma_{f r}$ is the tire workload of the right-front wheel, $\gamma_{r l}$ is the tire workload of left-rear wheel, and $\gamma_{r r}$ is the tire workload of right-rear wheel.

3.1.2. Optimized Torque-Distribution Control Based on Total Motor Efficiency. In order to improve the energy efficiency of the FREWL while transferring equipment, the objective function is that the total motor efficiency is maximal [12]. It can be defined as

$$
\max \quad \eta=\frac{T_{m} n}{2\left[T_{m f} / \eta_{f}\left(T_{m f}, n_{f}\right)+T_{m r} / \eta_{r}\left(T_{m r}, n_{r}\right)\right] n},
$$

where $\eta_{f}$ is the efficiency of front motor, $\eta_{r}$ is the efficiency of rear motor, $n_{f}$ is the front motor speed, and $n_{r}$ is the rear motor speed. The relationship between motor efficiency $\eta_{f}$, motor torque $T_{f}$, and motor speed $n_{f}$ is shown in Figure 3.

With (6), then (8) becomes

$$
\max \eta=\frac{1}{\kappa / \eta_{f}\left(T_{m f}, n_{f}\right)+(1-\kappa) / \eta_{r}\left(T_{m r}, n_{r}\right)} .
$$

3.2. Constraint Conditions. The total driving torque should satisfy expected accelerator position firstly, as is shown in

$$
T_{m}=f\left(\partial_{\text {pedal }}, n\right)
$$

where $\partial_{\text {pedal }}$ is the accelerator position and $n$ is the motor speed. The surface about the three parameters is shown as Figure 4. 


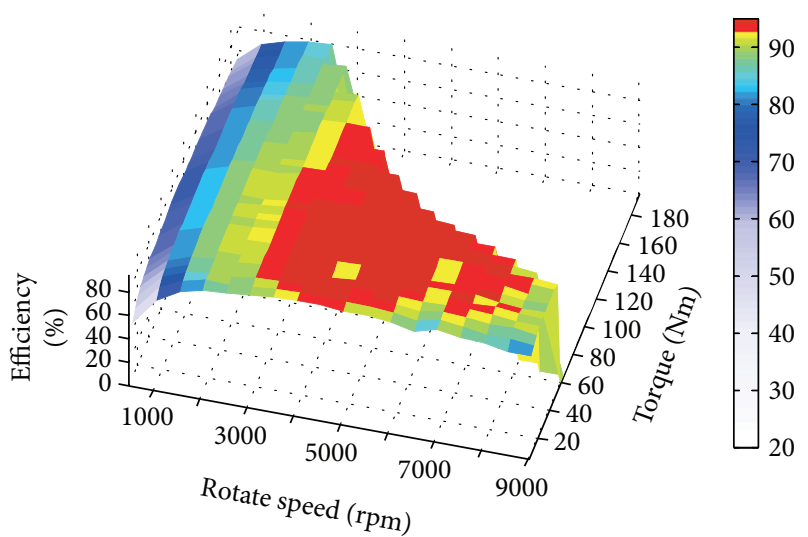

FIGURE 3: MAP diagram of motor.

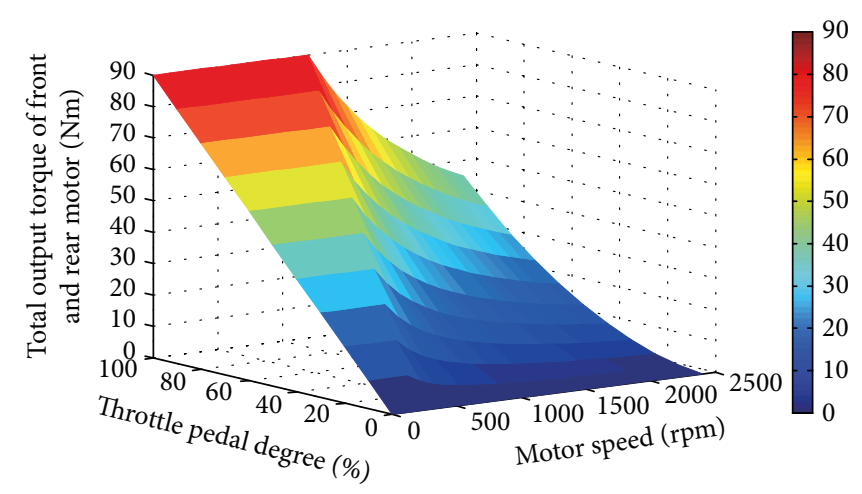

FIGURE 4: Defining surface of total driving torque of front/rear motor.

Adhesion force is influenced by wheel vertical load and tire-road friction coefficient. Because the influence of the motor inertia moment and wheel inertia moment is tiny, it is appropriate to ignore them. So in pure longitudinal slip condition, the maximum driving torque is limited by

$$
\left|T_{m i}\right| \leq \frac{2 \mu_{i, x} F_{i, z} r_{\mathrm{eff}}}{N} .
$$

The motor driving torque and speed should be limited as follows:

$$
\begin{aligned}
\left|T_{m i}\right| & \leq T_{\max } \\
n & \leq n_{\max } .
\end{aligned}
$$

The range of driving torque-distribution coefficient $\kappa$ is given by

$$
0 \leq \kappa \leq 1
$$

3.3. Optimization Algorithms. Nonlinear optimization algorithms are widely used to solve nonlinear optimization problems [28]. Each nonlinear optimization algorithm has its capabilities and limitations, which have a significant impact on the performance of OTCM. The common optimization algorithms for nonlinear constraints optimization problems are quasi-Newton Lagrangian multiplier method (QNLM), sequential quadratic programming (SQP), adaptive genetic algorithms (AGA), and particle swarm optimization with random weighting and natural selection, (PSO-RN). The notable advantage that these four algorithms possess over their classic one is the fast solution speed, which satisfies the requirement of subsequent field test of FREWL.

3.3.1. Quasi-Newton Lagrangian Multiplier Method. QuasiNewton method (QNM) is a special case of Newton method. The objective function is Taylor expanded in second order at $x_{k+1}$, as shown in (14).

$$
\begin{aligned}
f(x) \approx & f\left(x_{k+1}\right)+g_{k+1}^{T}\left(x-x_{k+1}\right) \\
& +\frac{1}{2}\left(x-x_{k+1}\right)^{T} G_{k+1}\left(x-x_{k+1}\right) .
\end{aligned}
$$

The derivative of (14) is

$$
g(x) \approx g_{k+1}+G_{k+1}\left(x-x_{k+1}\right) .
$$

An approximate matrix $B_{k}$ is used to replace $G_{k+1}$ in (15). The Broyden-Fletcher-Goldfarb-Shanno (BFGS) is used to update the $B_{k}$, as shown in (16).

$$
B_{k+1}=B_{k}-\frac{B_{k} s_{k} s_{k}^{T} B_{k}}{s_{k}^{T} B_{k} s_{k}}+\frac{y_{k} y_{k}^{T}}{y_{k}^{T} s_{k}} .
$$

Quasi-Newton method fails to solve the nonlinear constraints optimization problems. Thus Lagrangian multiplier (LM) is introduced into the problem. Inequality constraints are transformed into equality constraints by an auxiliary variable. Then with the original equality constraints, the Lagrangian function is transformed into

$$
\begin{aligned}
\psi(x, y, \lambda, \sigma) & \\
= & f(x)-\sum_{i=1}^{l} \mu_{i} h_{i}(x)+\frac{\sigma}{2} \sum_{i=1}^{l} h_{i}^{2}(x) \\
& +\frac{1}{2 \sigma} \sum_{i=1}^{m}\left(\left[\min \left\{0, \sigma g_{i}(x)-\lambda_{i}\right\}\right]^{2}-\lambda_{i}^{2}\right),
\end{aligned}
$$

where the iterative equation of the multiplier is

$$
\begin{aligned}
& \left(\mu_{k+1}\right)_{i}=\left(\mu_{k}\right)_{i}-\sigma h_{i}\left(x_{k}\right), \quad i=1,2, \ldots, l, \\
& \left(\lambda_{k+1}\right)_{i}=\max \left\{0,\left(\lambda_{k}\right)_{i}-\sigma g_{i}\left(x_{k}\right)\right\}, \quad i=1,2, \ldots, m .
\end{aligned}
$$

The termination criterion of iteration is

$$
\left(\sum_{i=1}^{l} h_{i}^{2}\left(x_{k}\right)+\sum_{i=1}^{m}\left[\min \left\{g_{i}\left(x_{k}\right), \frac{\left(\lambda_{k}\right)_{i}}{\sigma}\right\}\right]^{2}\right)^{1 / 2} \leq \varepsilon,
$$

where $\lambda$ is called a multiplier, $\sigma$ is penalty factor, and $\varepsilon$ is termination error ranging from 0 to 1 .

The simulation parameters of the QNLM used in this paper are shown in Table 2. QNLM consists of QNM and LM. QNM and LM have their own maximum iteration number and termination error. The maximum iteration and termination error have influence on the computing accuracy and computing time. Penalty factor $\sigma$ is used to penalize those individuals which do not satisfy the constraint condition for solving constrained optimization problems. 
TABLE 2: Simulation parameters of the QNLM.

\begin{tabular}{lc}
\hline Parameters & Values \\
\hline Maximum iteration number of QNM & 200 \\
Maximum iteration number of LM & 200 \\
Penalty factor of LM & 5 \\
Termination error of QNM & $1 e-5$ \\
Termination error of LM & $1 e-5$
\end{tabular}

3.3.2. Sequential Quadratic Programming. SQP is an efficient method for nonlinear optimization with advantages of high computational efficiency and fast convergent rate. In SQP, positive definite matrix $B_{0} \in R^{n \times n}$ is used to seek the optimal solution $d_{k}$ of subquestion. The subquestion is described as

$$
\begin{array}{ll}
\min & \frac{1}{2} d^{T} B_{k} d+\nabla f\left(x_{k}\right)^{T} d, \\
\text { s.t. } & h\left(x_{k}\right)+\nabla h\left(x_{k}\right)^{T} d=0, \\
& g\left(x_{k}\right)+\nabla g\left(x_{k}\right)^{T} d \geq 0 .
\end{array}
$$

If the constraints $\left\|d_{k}\right\|_{1} \leq \varepsilon_{1}$ and $\left\|h_{k}\right\|_{1}+\left\|\left(g_{k}\right)_{-}\right\|_{1} \leq \varepsilon_{2}$ are satisfied, the calculation will be determined. $\left[g_{k}(x)\right]_{-}=$ $\max \left\{0,-g_{k}(x)\right\}$, and 1 means the initial value. A point under Karush-Kuhn-Tucker (KKT) condition is obtained and the termination error is $0 \leq \varepsilon_{1}, \varepsilon_{2} \leq 1$.

To some value function $\phi(x, \sigma)$, the chosen penalty factor $\sigma$ defines that $d_{k}$ is decreasing at the point $x_{k}$. Suppose that $m_{k}$ is the tiniest nonnegative integer satisfying the following equation:

$$
\phi\left(x_{k}+\rho^{m} d_{k}, \sigma_{k}\right)-\phi\left(x_{k}, \sigma_{k}\right) \leq \eta \rho^{m} \phi^{\prime}\left(x_{k}, \sigma_{k} ; d_{k}\right),
$$

where $\eta \in(0,1 / 2)$ and $\rho \in(0,1)$.

From $\alpha_{k}=\rho^{m k}, x_{k+1}=x_{k}+\alpha_{k} d_{k}, A_{k+1}$ is given by

$$
A_{k+1}=\left(\nabla h\left(x_{k+1}\right)^{T}, \nabla g\left(x_{k+1}\right)^{T}\right)^{T} .
$$

The least square multiplier is calculated by

$$
\left(\begin{array}{c}
\mu_{k+1} \\
\lambda_{k+1}
\end{array}\right)=\left[A_{k+1} A_{k+1}^{T}\right]^{-1} A_{k+1} \nabla f\left(x_{k+1}\right) .
$$

The approximate matrix $B_{k}$ is the same as in (16).

The simulation parameters of the SQP used in this paper are shown in Table 3. SQP needs to solve a quadratic programming subproblem at every iteration step. It is necessary to set its and subproblem's maximum iteration number and termination error. The values of maximum iteration number, termination error, and penalty factor are the same as the function in QNLM.

3.3.3. Adaptive Genetic Algorithms. AGA is another significant and promising variant of genetic algorithms. AGA adjusts probabilities of crossover and probabilities of mutation in order to maintain the genetic model and to accelerate the convergence speed. In AGA, the evolution usually starts
TABLE 3: Simulation parameters of SQP.

\begin{tabular}{lc}
\hline Parameters & Values \\
\hline Maximum iteration number & 200 \\
Iteration number of subproblem & 200 \\
Penalty factor & 0.05 \\
Termination error & $1 e-5$ \\
Termination error of subproblem & $1 e-5$ \\
\hline
\end{tabular}

TABLE 4: Simulation parameters of the AGA.

\begin{tabular}{lc}
\hline Parameters & Values \\
\hline Lower bound of independent variable & 1 \\
Upper bound of independent variable & 0 \\
Scale of population & 50 \\
Maximum evolution generations & 200 \\
Discrete precision of independent variable & $1 e-5$ \\
Crossover constant $k_{1}$ & 0.5 \\
Crossover constant $k_{2}$ & 0.9 \\
Mutation constant $k_{3}$ & 0.03 \\
Mutation constant $k_{4}$ & 0.07
\end{tabular}

from a population which consisted of randomly generated individuals. By Roulette strategy, individual fitness values are evaluated to judge if it agrees with the optimization criterion. The new individuals are generated by the optimal best mutation probability $P_{m}$ and the best crossover probability $P_{c}$ whose equations are shown in

$$
\begin{gathered}
P_{c}= \begin{cases}\frac{k_{1}\left(f_{\max }-f\right)}{f_{\max }-f_{\mathrm{avg}}}, & f \geq f_{\mathrm{avg}} \\
k_{2}, & f<f_{\mathrm{avg}}\end{cases} \\
P_{m}= \begin{cases}\frac{k_{3}\left(f_{\max }-f^{\prime}\right)}{f_{\max }-f_{\text {avg }}}, & f^{\prime} \geq f_{\mathrm{avg}} \\
k_{4}, & f^{\prime}<f_{\text {avg }},\end{cases}
\end{gathered}
$$

where $f_{\max }$ is the maximum fitness value population, $f_{\text {avg }}$ is the mean fitness value population, $f$ is the bigger fitness value in two individuals about to crossover, and $f^{\prime}$ is the fitness value of individuals about to mutate. $k_{1}, k_{2}, k_{3}$, and $k_{4}$ are the constants.

The simulation parameters of AGA used in this paper are shown in Table 4. Among them, the lower bound and upper bound of independent variable and discrete precision determine the encoding length required for the binary encoding. The values for lower bound and upper bound depend on the constraints. The scale of population and the maximum ecology affect the accuracy and computing time. In order to analyze the advantages and disadvantages of the various algorithms as much as possible, the maximum evolution generations are the same with the maximum number of the same number of QNLM and SQP. The values of $k_{1}, k_{2}, k_{3}$, and $k_{4}$ are usually based on different application objects. It generally requires that $k_{1}<k_{2}, k_{3}<k_{4}$. 
TABLE 5: Simulation parameters of the PSO-RN.

\begin{tabular}{lc}
\hline Parameters & Values \\
\hline Particle population & 30 \\
Acceleration constant $c_{1}$ & 2 \\
Acceleration constant $c_{2}$ & 2 \\
Upper boundary of inertia weight & 0.9 \\
Lower boundary of inertia weight & 0.4 \\
Maximum evolution generations & 100 \\
Dimension of search space & 1 \\
Maximum particle velocity $v_{\max }$ & 0.2 \\
Minimum particle velocity $v_{\min }$ & 0 \\
Maximum particle position $x_{\max }$ & 1 \\
Minimum particle position $x_{\min }$ & 0 \\
\hline
\end{tabular}

3.3.4. Particle Swarm Optimization with Random Weighting and Natural Selection. Particle swarm optimization (PSO) based on natural selection is one of the improved algorithms, which is characterized by iteratively trying to improve a candidate solution. In each iteration, the worst half of the particles in the population is replaced by the best half of the particles while preserving the original historical optimal value. Therefore, it improves the optimization ability and solving speed and significantly reduces the algorithm premature convergence situation.

The inertia weight $\alpha$ is an important parameter in the PSO which is used to control the ability of development and search. The core of avoiding falling into the local optimal is to determine a reasonable inertia weight. In order to accelerate up the convergence speed, the inertia weight $\alpha$ is set as a random value. The equation to calculate the random $\alpha$ is

$$
\begin{aligned}
& \omega=\alpha+\sigma \times N(0,1) \\
& \alpha=\alpha_{\text {min }}+\left(\alpha_{\text {max }}-\alpha_{\text {min }}\right) \times \operatorname{rand}(0,1),
\end{aligned}
$$

where $\sigma$ is the random weight, $\alpha_{\max }$ and $\alpha_{\min }$ are the maximum and minimum of the inertia weight, and $N(0,1)$ is the random number of standard state distribution.

The simulation parameters of the PSO-RN used in this paper are shown in Table 5. Acceleration constants $c_{1}$ and $c_{2}$ determine the influence of particle individual experience and group experience on the trajectory of particle movement, usually $c_{1}=c_{2}$. Inertia weight can be used to control the algorithm development and search capabilities, which have different values according to different application problems. Dimension of search space has the same value as the number of its independent variables. Maximum particle position $x_{\max }$ and minimum particle position $x_{\min }$ are the boundary conditions of the algorithm, and the numerical value depends on the constraints of the actual problem. Maximum particle velocity $v_{\max }$ determines the maximum distance that the particle can move in one flight, usually $v_{\max }=k x_{\max }, 0.1 \leq$ $k \leq 1$.
TABLE 6: Simulation parameters based on tire workload.

\begin{tabular}{lccc}
\hline & Condition 1 & Condition 2 & Condition 3 \\
\hline$\mu_{f}$ & 0.8 & 0.2 & 0.2 \\
$\mu_{r}$ & 0.3 & 0.7 & 0.2 \\
$T_{m}(\mathrm{Nm})$ & 300 & 300 & 360 \\
$F_{x}(\mathrm{~N})$ & - & 600 & 600 \\
$\alpha\left(^{\circ}\right)$ & - & - & 20 \\
\hline
\end{tabular}

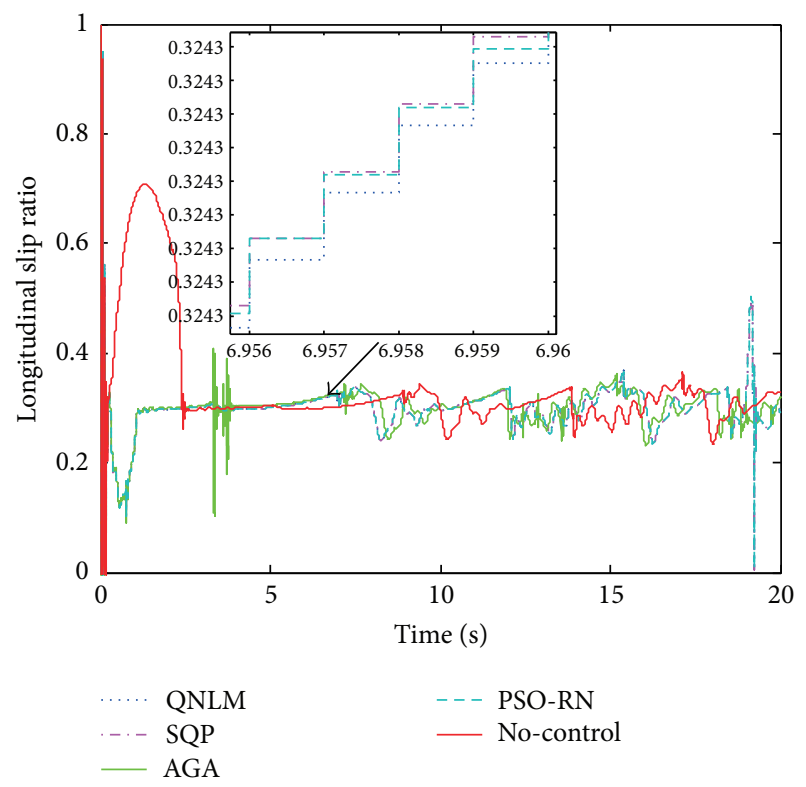

Figure 5: Longitudinal slip ratio of front wheel.

\section{Simulation Results}

4.1. Simulation of OTCM Based on Tire Workload. Conditions of traveling, spading, and stacking on bumpy road are common for wheel loader. The paper establishes these three conditions to verify the improvement of the FREWL operation performance through OTCM based on tire workload. Condition 1 simulates the traveling condition, while condition 2 simulates the spading condition. Condition 3 simulates the stacking condition in bumpy road with $20^{\circ}$ slope. The simulation parameters are listed in Table 6 .

From Figures 5 and 6 , it is notable that in the first $2 \mathrm{~s}$, namely, the starting stage, the longitudinal slip ratio of controlled FREWL is much smaller than no-control FREWL. The operation performance has significantly improved by OTCM. After $3 \mathrm{~s}$, namely, the driving stage, the longitudinal ratio optimized by AGA is smoother than other three algorithms, so the operation performance of FREWL is the best.

Another parameter to evaluate the control effect is the driving distance of the controlled FREWL and no-control FREWL on bumpy road in the same time, which is shown in Figure 7. Table 7 shows the driving distance of the FREWL without spading. Controlled FREWL has better control to longitudinal slip ratio; thus it marches earlier and drives farther than no-control FREWL. The OTCM based on AGA has the best control effect, and the driving distance compared 


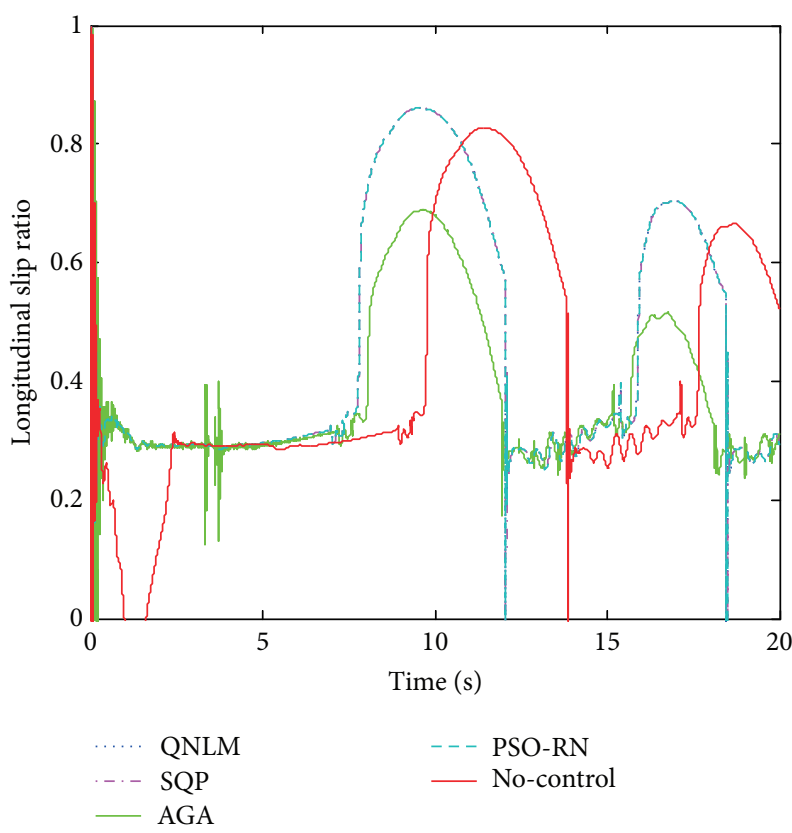

FIGURE 6: Longitudinal slip ratio of rear wheel.

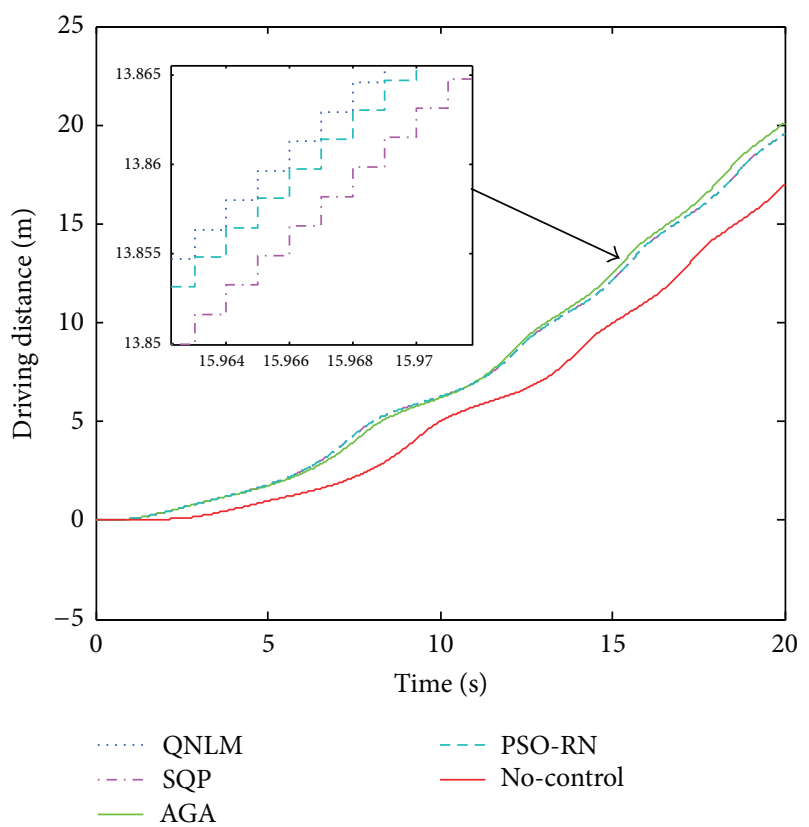

FIGURE 7: Driving distance in condition 1.

TABLE 7: Driving distance without spading resistance.

\begin{tabular}{lcc}
\hline & Final distance $(\mathrm{m})$ & Distance increase $(\%)$ \\
\hline No-control & 16.92 & - \\
SQP & 19.53 & 15.42 \\
AGA & 20.08 & 18.68 \\
PSO-RN & 19.54 & 15.48 \\
QNLM & 19.54 & 15.48 \\
\hline
\end{tabular}

to no-control FREWL is increased by $18.68 \%$. The control effect of other three optimization algorithms is basically the

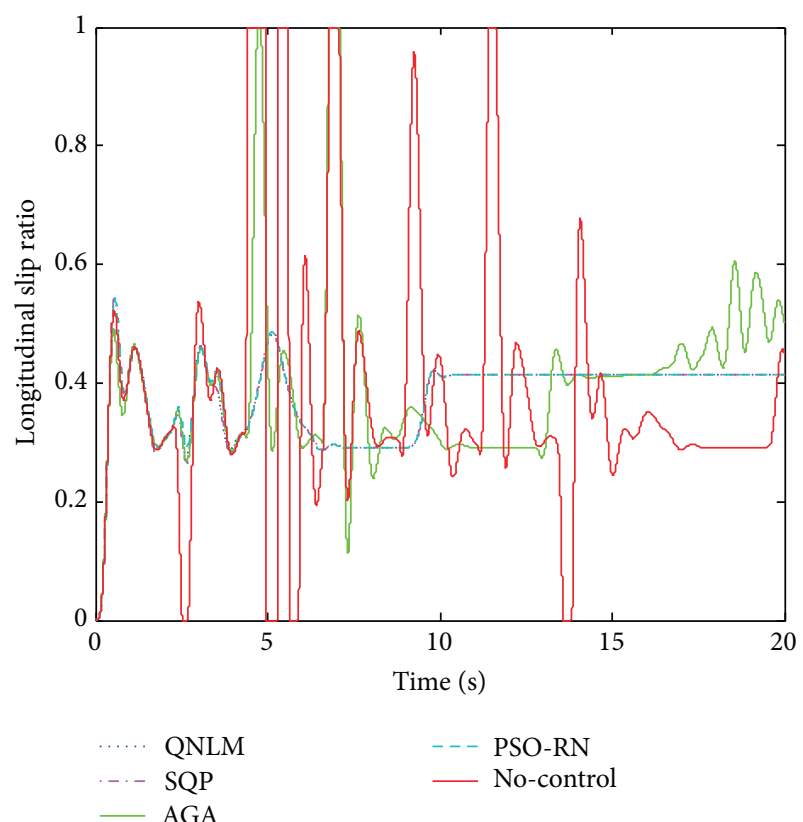

FIGURE 8: Longitudinal slip ratio of front wheel.

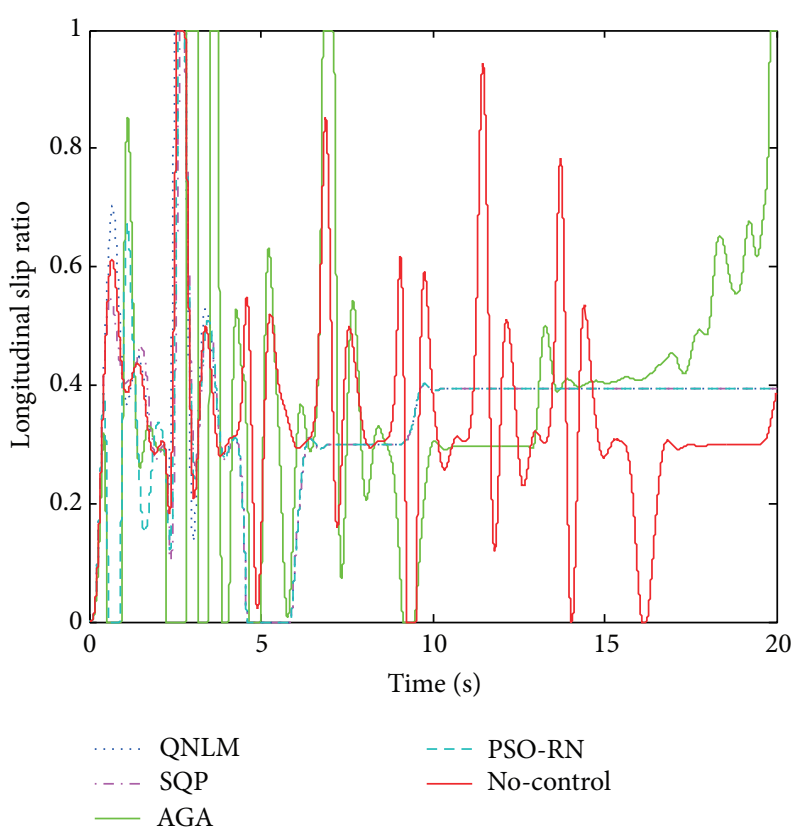

FIGURE 9: Longitudinal slip ratio of rear wheel.

same; the driving distance is increased by $15.48 \%$ compared to the no-control FREWL.

Figures 8 and 9 show the longitudinal slip ratio of front and rear wheel in condition 2, respectively. Table 8 shows slip frequency of the front and rear wheel of the controlled FREWL and no-control FREWL. In whole simulation time, especially the starting stage, the control effect of OTCM based on SQP is the best. Only the rear wheel slips once.

Figure 10 shows the driving distance of FREWL in condition 2. Controlled FREWL utilizes adhesion ability better 
TABLE 8: Slip frequency of the front and rear wheels.

\begin{tabular}{lcc}
\hline & Front wheel slip times & Rear wheel slip times \\
\hline No-control & 4 & 1 \\
SQP & 0 & 1 \\
AGA & 2 & 4 \\
PSO-RN & 0 & 1 \\
QNLM & 0 & 1 \\
\hline
\end{tabular}

TABLE 9: Forward driving time with spading resistance.

\begin{tabular}{lcc}
\hline & Driving time $(\mathrm{s})$ & Time decrease $(\%)$ \\
\hline No-control & 15.42 & - \\
SQP & 3.98 & 74.19 \\
AGA & 8.42 & 45.39 \\
PSO-RN & 3.92 & 74.59 \\
QNLM & 3.96 & 74.32
\end{tabular}

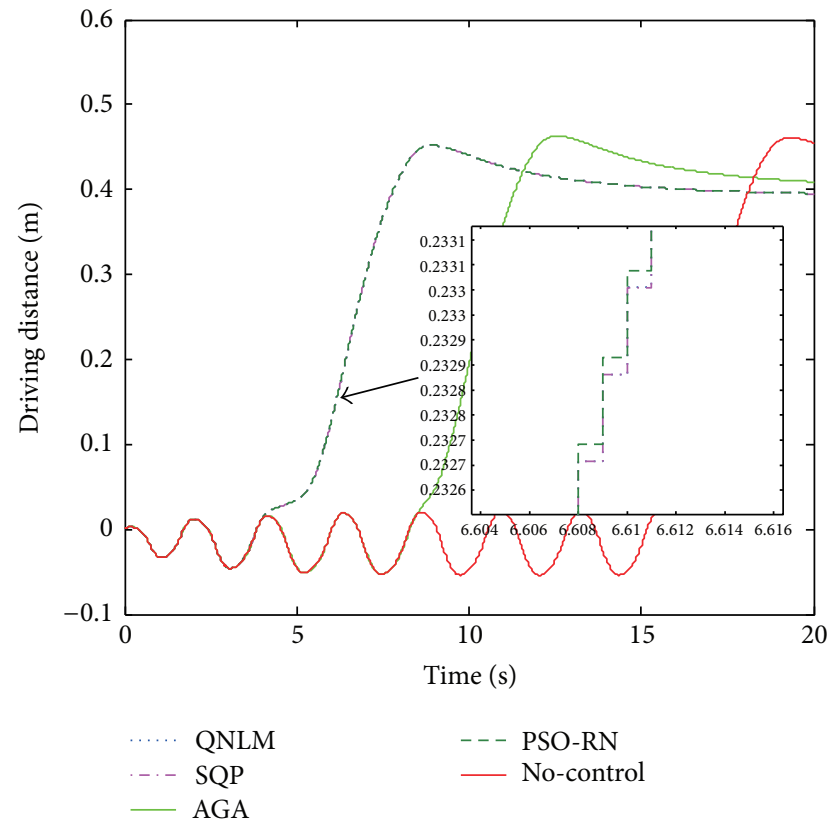

FIGURE 10: Driving distance in condition 2.

and slips less and also can go forward in a shorter period when encountering spading resistance, as shown in Table 9. In this case, FREWL controlled by QNLN, SQP, and PSO$\mathrm{RN}$ can go forward in a shorter period and make significant improvement, but the control effect of OTCM based on AGA is undesirable.

Condition 3 simulates stacking condition that the FREWL operates on the bumpy road with $20^{\circ}$ slope and encounters a continuous spading resistance after $5 \mathrm{~s}$, and is gradually heavy-loaded. Compared to conditions 1 and 2, condition 3 is more complicated. Figures 11 and 12 show the longitudinal slip ratio of front and rear wheel in condition 3. It is obvious that after $10 \mathrm{~s}$ the front wheel of no-control FREWL is basically in the slippery state. While the FREWL applies four optimization algorithms, the slippery time is much less.
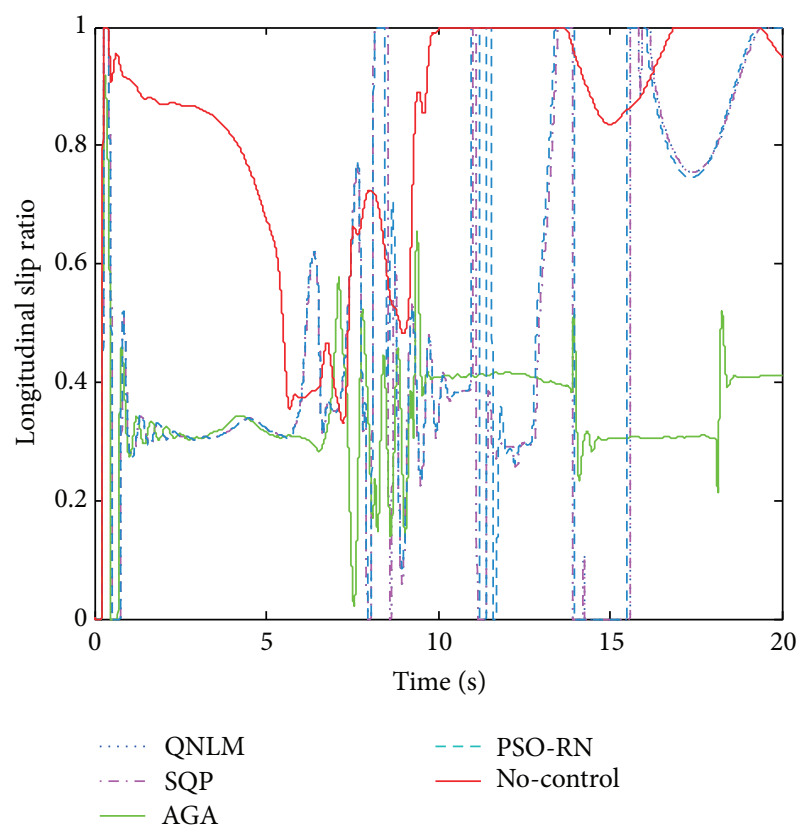

FIGURE 11: Longitudinal slip ratio of front wheel.

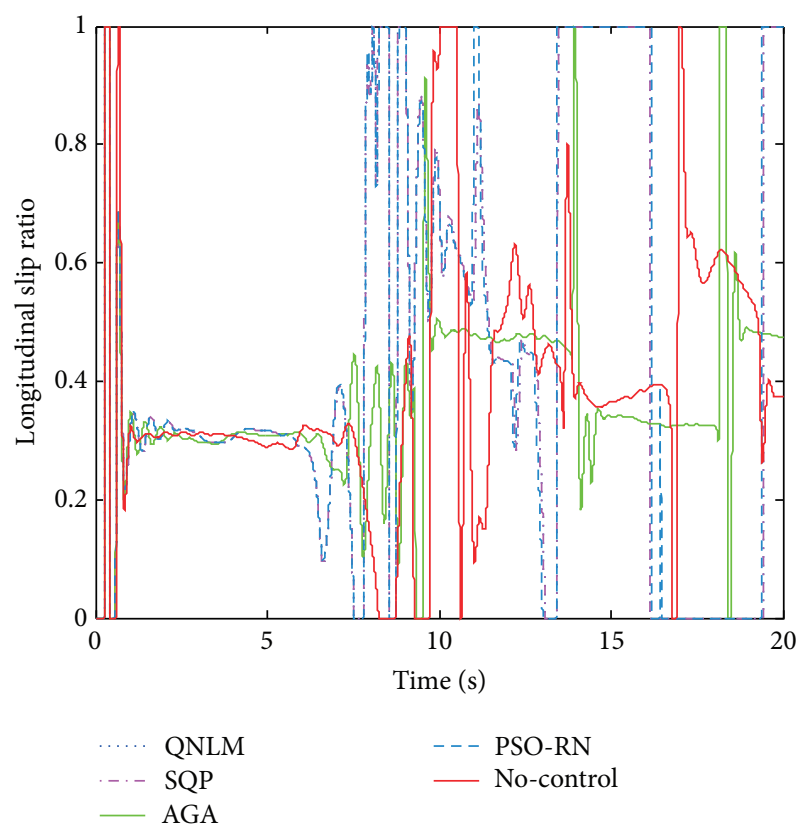

FIGURE 12: Longitudinal slip ratio of rear wheel.

Among them, AGA-controlled FREWL slips the least. The control effects of the other three algorithms are similar.

Figure 13 shows the driving distance of FREWL in condition 3. Since AGA is better in control of the longitudinal slip ratio of front/rear wheel in Figures 11 and 12, it drives farthest. The other three algorithms also have a good performance compared to no-control. This shows that FREWL, which applied OTCM based on tire workload, has better operation performance in stacking condition than no-control FREWL. 


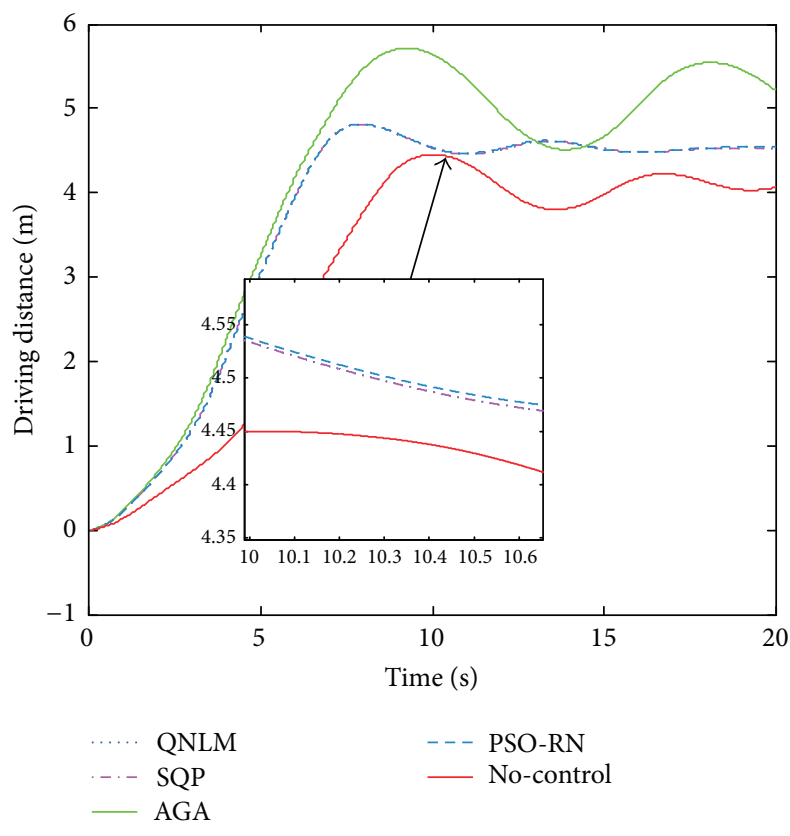

FIGURE 13: Driving distance in condition 3.

TABLE 10: Simulation parameters based on total motor efficiency.

\begin{tabular}{lcc}
\hline & Condition 4 & Condition 5 \\
\hline Motion state & Straight driving & Reciprocating driving \\
$\mu_{f}$ & 0.8 & 0.6 \\
$\mu_{r}$ & 0.8 & 0.6 \\
$v_{0}(\mathrm{~m} / \mathrm{s})$ & 0 & 10 \\
$T_{m}(\mathrm{Nm})$ & 100 & 200 \\
\hline
\end{tabular}

TABLE 11: Total motor efficiency in straight driving.

\begin{tabular}{lcc}
\hline & $\begin{array}{c}\text { Maximum total } \\
\text { motor efficiency }(\%)\end{array}$ & $\begin{array}{c}\text { Efficiency increase } \\
(\%)\end{array}$ \\
\hline No-control & 43.63 & - \\
SQP & 49.58 & 13.64 \\
AGA & 49.57 & 13.48 \\
PSO-RN & 50.12 & 14.86 \\
\hline
\end{tabular}

\subsection{Optimized Torque-Distribution Control Based on Total} Motor Efficiency. Two most common equipment transferring conditions are driving straightly on the bituminous road and reciprocating on bumpy road. This paper sets two conditions to simulate the improvement of energy efficiency of FREWL by OTCM while transferring equipment. The simulation parameters are listed in Table 10. Condition 4 is set to observe the energy efficiency of FREWL while driving straightly on the bituminous road. Condition 5 is set to observe the energy efficiency of FREWL while reciprocating on bumpy road.

Figure 14 shows the total motor efficiency of the FREWL when the FREWL is traveling on the bituminous road. Table 11 shows the maximum motor efficiency of the various optimization algorithms. The total motor efficiency optimized by OTCM based on QNLM fluctuates frequently

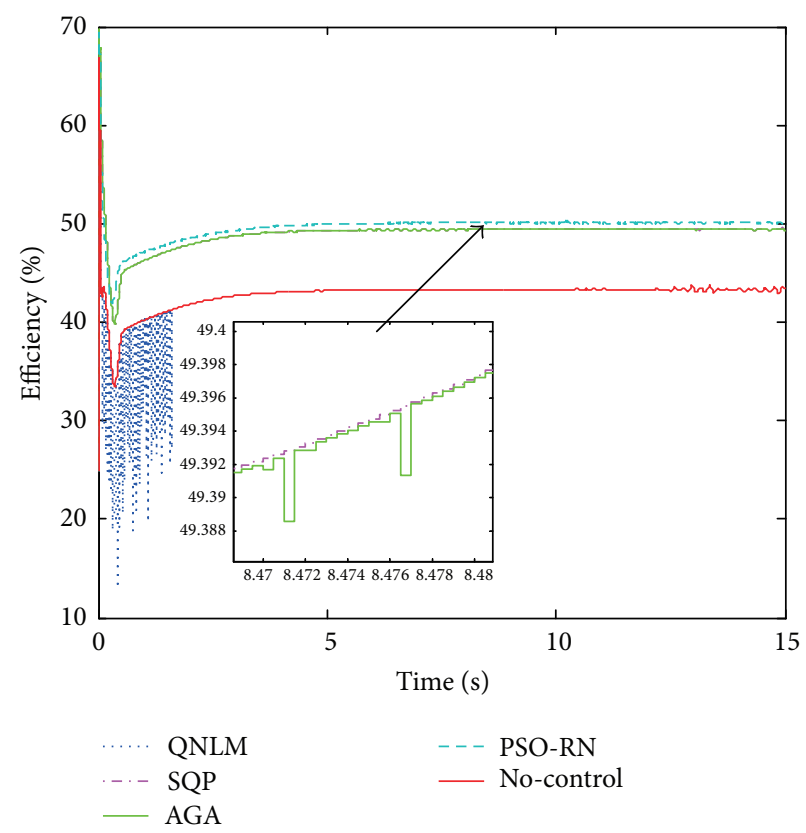

FIgURE 14: Total motor efficiency in condition 4.

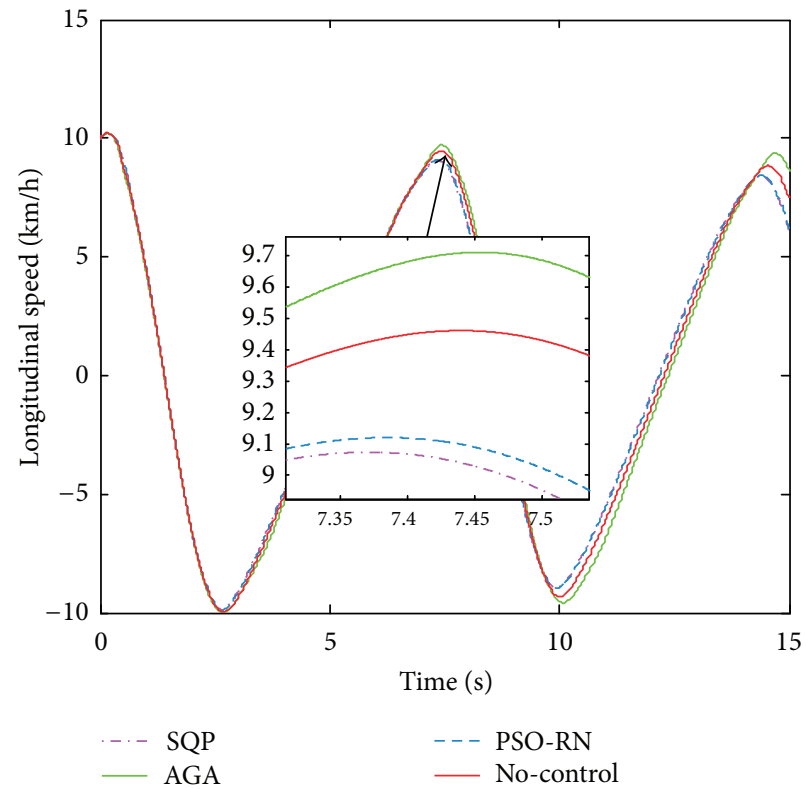

FIgURE 15: Longitudinal speed in condition 5.

and violently in the starting stage of simulation, which leads to calculation failure, which means that QNLM is not suitable for problems with strong-nonlinearity. The results of other three optimization algorithms are better than no-control FREWL. The PSO-RN works best; the total motor efficiency is increased by $14.86 \%$ comparing to no-control FREWL. The results of SPQ and AGA are roughly the same; the total motor efficiency is increased by $13.64 \%$ comparing to no-control FREWL.

Figures 15 and 16 show longitudinal speed and total motor efficiency of FREWL in condition 5 . Table 12 shows the maximum total motor efficiency of FREWL in the forward 
TABLE 12: Total motor efficiency in reciprocating driving.

\begin{tabular}{lcccc}
\hline & $\begin{array}{c}\text { Forward-total motor } \\
\text { efficiency }(\%)\end{array}$ & $\begin{array}{c}\text { Forward-efficiency } \\
\text { increase (\%) }\end{array}$ & $\begin{array}{c}\text { Backward-total motor } \\
\text { efficiency (\%) }\end{array}$ & $\begin{array}{c}\text { Backward-efficiency } \\
\text { increase (\%) }\end{array}$ \\
\hline No-control & 46.53 & - & 28.61 & - \\
SQP & 53.62 & 15.24 & 36.02 & 25.90 \\
AGA & 51.68 & 11.07 & 36.94 & 29.12 \\
PSO-RN & 52.25 & 12.29 & 34.52 & 20.66 \\
\hline
\end{tabular}

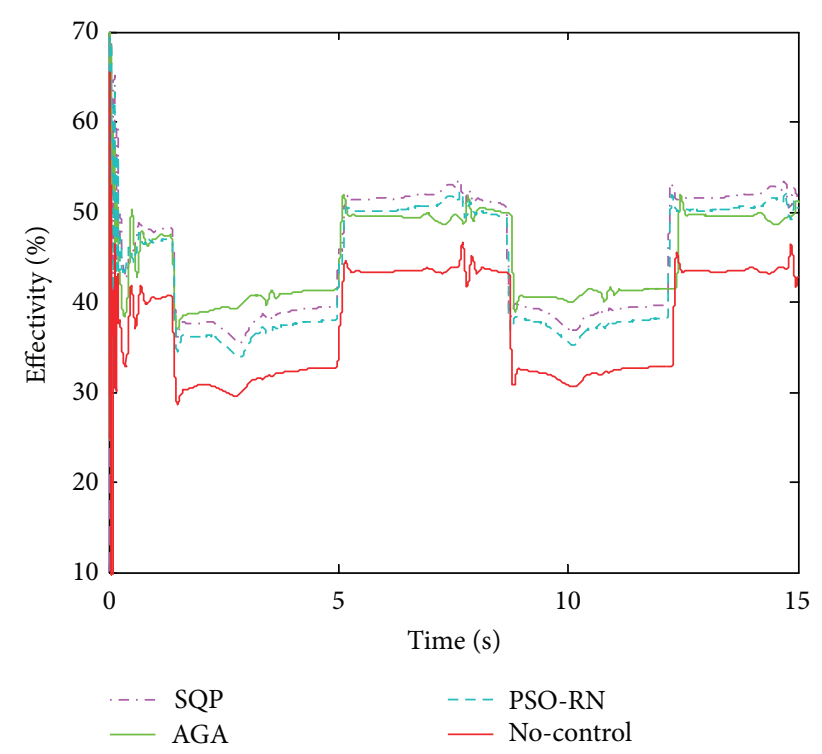

FIGURE 16: Total motor efficiency in condition 5.

stage and the backward stage. The total motor efficiency of controlled FREWL is greatly improved compared to nocontrol FREWL when driving reciprocally. The results by OTCM perform better in backward stage than in forward stage. The solution of SQP is the best in forward stage; the total motor efficiency is increased by $15.24 \%$ at most. The result of AGA works best in backward stage; the total motor efficiency is increased by $29.12 \%$ at most.

4.3. Simulation Time. Simulation time is an essential factor to affect the practicability of the method. Table 13 shows the simulation time of the optimization algorithms under various conditions. The simulation step size is $0.001 \mathrm{~s}$ in the Simulink/Carsim platforms. Taking into account the actual real test, the sampling step is generally set to $0.01 \mathrm{~s}$. Therefore, QNLM and SQP can be used in the online control, but AGA and PSO-RN cannot. Because QNLM fails in equipment transferring condition, SQP is comprehensively the best optimization algorithm for OTCM in actual test.

\section{Discussion}

In this paper, we study the OTCM of FREWL and prove that OTCM can improve the operation performance and energy efficiency of FREWL through five simulation conditions. In addition to five simulation conditions mentioned in this
TABLE 13: Simulation time of OTCM on different conditions.

\begin{tabular}{lcccc}
\hline & QNLM (s) & SQP (s) & AGA (min) & PSO-RN (min) \\
\hline Condition 1 & 32.7 & 55.6 & 69.2 & 27.3 \\
Condition 2 & 23.5 & 36.1 & 88.3 & 54.8 \\
Condition 3 & 32.1 & 41.6 & 64.9 & 29.5 \\
Condition 4 & - & 17.9 & 52.7 & 15.7 \\
Condition 5 & - & 13.9 & 54.7 & 18.1 \\
\hline
\end{tabular}

TABLE 14: Parameters changed in other conditions.

\begin{tabular}{lccccc}
\hline & $m$ & $\alpha$ & $\dot{v}_{x}$ & $F_{x}$ & $F_{z}$ \\
\hline Heavy-haul transportation & $\checkmark$ & - & $\checkmark$ & - & - \\
Loading/unloading material & $\checkmark$ & - & $\checkmark$ & $\checkmark$ & $\checkmark$ \\
Bulldozing & $\checkmark$ & - & $\checkmark$ & $\checkmark$ & $\checkmark$ \\
Climbing & - & $\checkmark$ & $\checkmark$ & - & - \\
\hline
\end{tabular}

paper, this method is also adaptable to other operation and equipment transferring conditions of FREWL in longitudinal motion. The changed parameters are shown in Table 14. Tick " $\checkmark$ " means this parameter is changed in that situation and hyphen "- " means it is unchanged.

As can be seen from Table 14, in other conditions, all the changed parameters are taken into account in the dynamic model of FREWL. At the same time, the optimization algorithm and optimization goals do not change. Therefore, the method proposed in this paper is suitable for operation and equipment transferring conditions.

In the simulation case, compared to no-control FREWL, the FREWL controlled by four optimization algorithms have a great increase in the operation performance and energy efficiency. In the OTCM based on tire workload, the QNLM and SQP optimization solutions are almost identical, because their core algorithm is the BFGS algorithm, as shown in (16). PSO-RN and AGA are modern intelligent optimization algorithms, but their results are quite different because the optimal solution of PSO-RN is easy to fall into the local optimal solution. Comparing to PSO-RN, AGA increases the crossover probability and mutation probability when the optimal solution tends to local optimal solution, enhancing the ability to solve the global optimal solution. Thus, AGA generally has a better performance in most simulation conditions.

However, although the AGA has a better performance, it has the longest computing time because the movement of the whole population is more evenly moving to the optimal region. QNLM and SQP compute faster because they apply 
the traditional BFGS method. Among them, SQP needs to solve a quadratic programming subproblem at each iteration step, so the calculation time is longer than QNLM.

\section{Conclusion}

OTCM is a critical technology to improve the operation performance and energy efficiency of FREWL. The driving torque of front motor and rear motor of FREWL can be controlled independently. The objective function minimizes the weighted sum of variance and mean value of tire workload and maximizes the total motor efficiency. The results show that the operation performance and energy efficiency are obviously improved by OTCM. While the FREWL is operating, the frequency of slip is obviously reduced, and the adhesion ability is improved. While the FREWL is driving straightly in equipment transferring, total motor efficiency is improved by $14.86 \%$ at most. While the FREWL is driving reciprocally in equipment transferring, total motor efficiency is improved by $29.12 \%$ at most. Considering the simulation results and simulation time comprehensively, SQP is the most suitable one of the four optimization algorithms for field test of FREWL.

\section{Conflicts of Interest}

The authors declare that there are no conflicts of interest regarding the publication of this paper.

\section{Acknowledgments}

The authors acknowledge the funding support from National Natural Science Foundation of China, no. 51375202, and also acknowledge the support of Programs for Science Research and Development of Jilin Province, no. 20160101285JC.

\section{References}

[1] Z. Naiwei, D. Qunliang, J. Yuanhua, Z. Wei, and R. Youcun, "Modeling and simulation research of coaxial parallel hybrid loader," Applied Mechanics and Materials, vol. 29-32, pp. 16341639, 2010.

[2] J. Wang, Z. Yang, S. Liu, Q. Zhang, and Y. Han, "A comprehensive overview of hybrid construction machinery," Advances in Mechanical Engineering, vol. 8, no. 3, pp. 1-15, 2016.

[3] F. Wang, M. A. Mohd Zulkefli, Z. Sun, and K. A. Stelson, "Energy management strategy for a power-split hydraulic hybrid wheel loader," Proceedings of the Institution of Mechanical Engineers, Part D: Journal of Automobile Engineering, vol. 230, no. 8, pp. 1105-1120, 2016.

[4] P. Song, M. Tomizuka, and C. Zong, "A novel integrated chassis controller for full drive-by-wire vehicles," Vehicle System Dynamics, vol. 53, no. 2, pp. 215-236, 2015.

[5] Y. Dai, Y. Luo, W. Chu, and K. Li, "Optimum tyre force distribution for four-wheel-independent drive electric vehicle with active front steering," International Journal of Vehicle Design, vol. 65, no. 4, pp. 336-359, 2014.

[6] H. Fujimoto and K. Maeda, "Optimal yaw-rate control for electric vehicles with active front-rear steering and four-wheel driving-braking force distribution," in Proceedings of the 39th
Annual Conference of the IEEE Industrial Electronics Society (IECON '13), pp. 6514-6519, IEEE, Vienna, AUSTRIA, November 2013.

[7] J. Kim, "Optimal power distribution of front and rear motors for minimizing energy consumption of 4 -wheel-drive electric vehicles," International Journal of Automotive Technology, vol. 17, no. 2, pp. 319-326, 2016.

[8] A. Pennycott, L. De Novellis, A. Sabbatini, P. Gruber, and A. Sorniotti, "Reducing the motor power losses of a fourwheel drive, fully electric vehicle via wheel torque allocation," Proceedings of the Institution of Mechanical Engineers, Part D: Journal of Automobile Engineering, vol. 228, no. 7, pp. 830-839, 2014.

[9] T. Cheong, S. H. Song, and C. Hu, "Strategic alliance with competitors in the electric vehicle market: tesla motor's case," Mathematical Problems in Engineering, vol. 2016, Article ID 7210767, 10 pages, 2016.

[10] H. He, J. Fan, Y. Li, and J. Li, "When to switch to a hybrid electric vehicle: a replacement optimisation decision," Journal of Cleaner Production, vol. 148, pp. 295-303, 2017.

[11] Y. Suzuki, Y. Kano, and M. Abe, "A study on tyre force distribution controls for full drive-by-wire electric vehicle," Vehicle System Dynamics, vol. 52, no. 1, pp. 235-250, 2014.

[12] Z.-P. Yu, L.-J. Zhang, and L. Xiong, "Optimized torque distribution control to achieve higher fuel economy of $4 \mathrm{WD}$ electric vehicle with four in-wheel motors," Journal of Tongji University, vol. 33, no. 10, pp. 79-85, 2005.

[13] D. Wang and B. Wang, "Research on driving force optimal distribution and fuzzy decision control system for a dual-motor electric vehicle," in Proceedings of the 34th Chinese Control Conference (CCC '15), pp. 8146-8153, IEEE, Hangzhou, China, July 2015

[14] A. F. Izmailov, M. V. Solodov, and E. I. Uskov, "Globalizing stabilized sequential quadratic programming method by smooth primal-dual exact penalty function," Journal of Optimization Theory and Applications, vol. 169, no. 1, pp. 148-178, 2016.

[15] M. A. Z. Raja, A. Zameer, A. U. Khan, and A. M. Wazwaz, "A new numerical approach to solve Thomas-Fermi model of an atom using bio-inspired heuristics integrated with sequential quadratic programming," SpringerPlus, vol. 5, no. 1, article 1400, 2016.

[16] J. Apolinar and M. Rodríguez, “Three-dimensional microscope vision system based on micro laser line scanning and adaptive genetic algorithms," Optics Communications, vol. 385, pp. 1-8, 2017.

[17] B. Bao, Y. Yang, Q. Chen, A. Liu, and J. Zhao, "Task allocation optimization in collaborative customized product development based on double-population adaptive genetic algorithm," Journal of Intelligent Manufacturing, vol. 27, no. 5, pp. 1097-1110, 2016.

[18] Y. Chen and M. S. Li, "A harmonic parameter estimation method based on Particle Swarm Optimizer with Natural Selection," in Proceedings of the 1st International Conference on Information and Communication Technology Research (ICTRC '15), pp. 206-209, IEEE, Abu Dhabi, Arab Emipates, May 2015.

[19] Y. Hu, J. Lu, Y. Deng, and Z. Zhang, "MPPT algorithm based on particle swarm optimization with natural selection," in Proceedings of the 3rd International Conference on Machinery, Materials and Information Technology Applications (ICMMITA '15), pp. 1814-1817, Atlantis Press, Qingdao, China, November 2015.

[20] H. Inoue, "Introduction of PC200-8 hybrid hydraulic excavators," Report, Komatsu, vol. 54, no. 161, pp. 1-6, 2008. 
[21] M. Ochiai and S. Ryu, "Hybrid in construction machinery," in Proceedings of the 7th JFPS international symposium on fluid power, pp. 41-44, Toyama, Japan, September 2008.

[22] M. Ochiai, "Technical trend and problem in construction machinery," Construction Machinery, vol. 38, no. 4, pp. 20-24, 2002.

[23] S. Riyuu, M. Tamura, and M. Ochiai, "Hybrid construction machine," Patent 2003328397, Japan, 2003.

[24] J. Deere, "644K Hybrid Wheel Loader," 2017, http://www.deere .com/wps/dcom/en_US/products/equipment/wheel_loaders/ $644 \mathrm{k}$ hybrid/644k_hybrid.page.

[25] J. Global, "L-950 Wheel Loader," 2017, https://mining.komatsu/ product-details/l-950\#!technology.

[26] S. Hui and J. Junqing, "Research on the system configuration and energy control strategy for parallel hydraulic hybrid loader," Automation in Construction, vol. 19, no. 2, pp. 213-220, 2010.

[27] N. Zhou, E. Zhang, Q. Wang et al., "Compound hybrid wheel loader," Patent CN102653228A, China, 2012.

[28] M. P. Aghababa, "3D path planning for underwater vehicles using five evolutionary optimization algorithms avoiding static and energetic obstacles," Applied Ocean Research, vol. 38, pp. 48-62, 2012. 


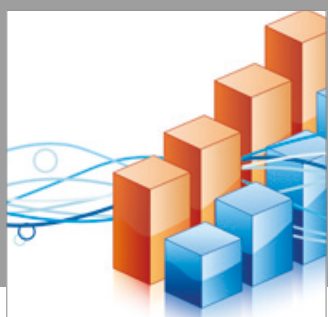

Advances in

Operations Research

vatersals

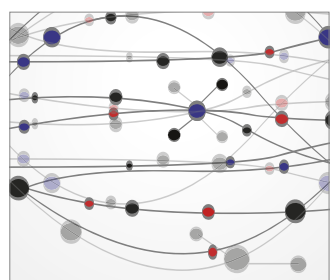

\section{The Scientific} World Journal
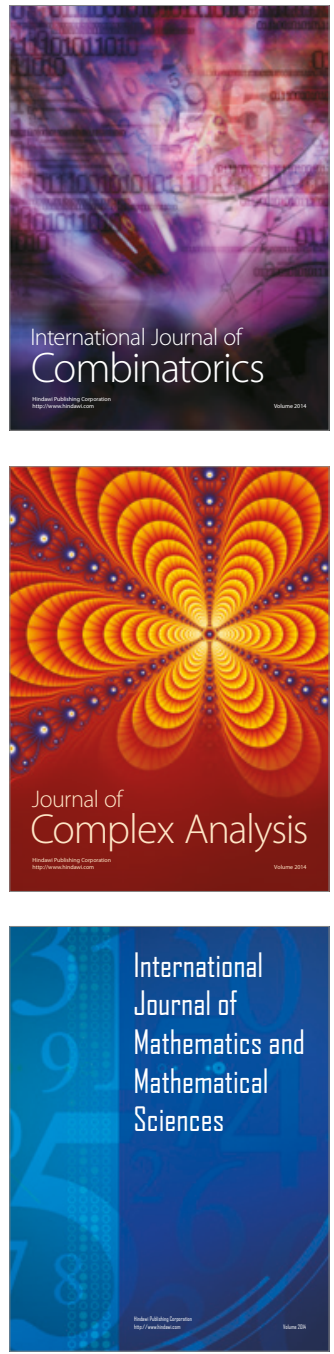
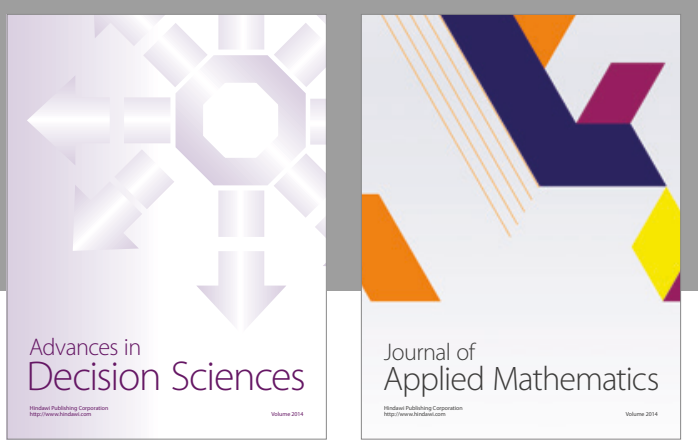

Algebra

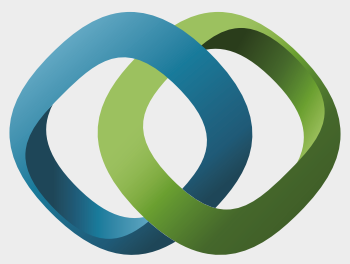

\section{Hindawi}

Submit your manuscripts at

https://www.hindawi.com
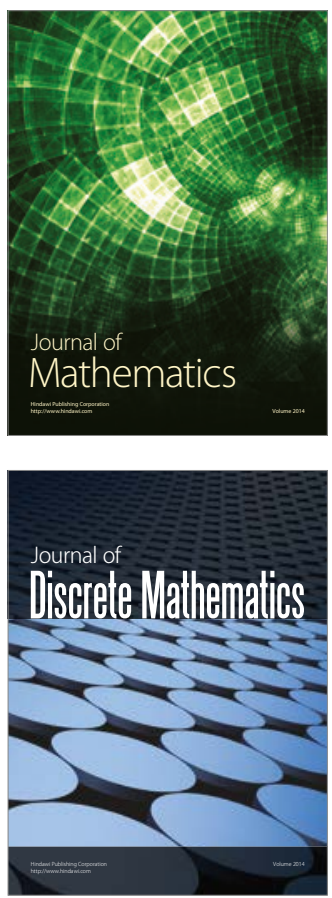

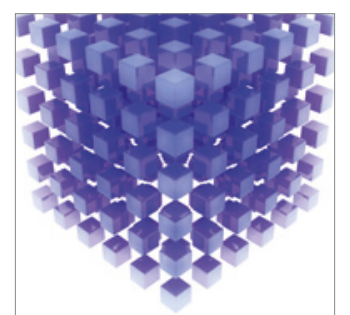

Mathematical Problems in Engineering
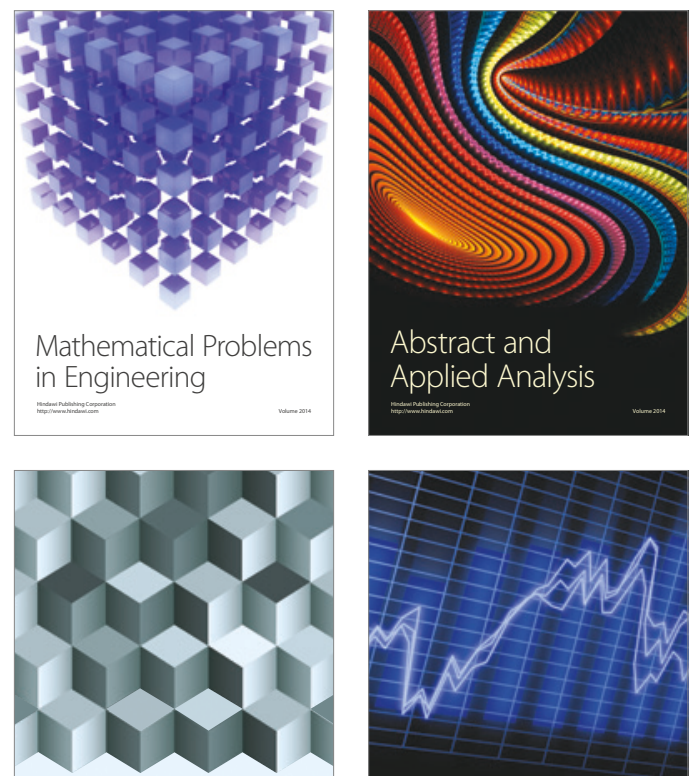

Journal of

Function Spaces

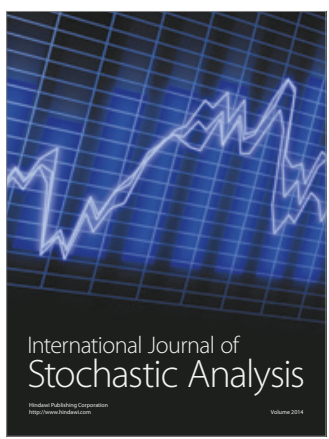

Probability and Statistics
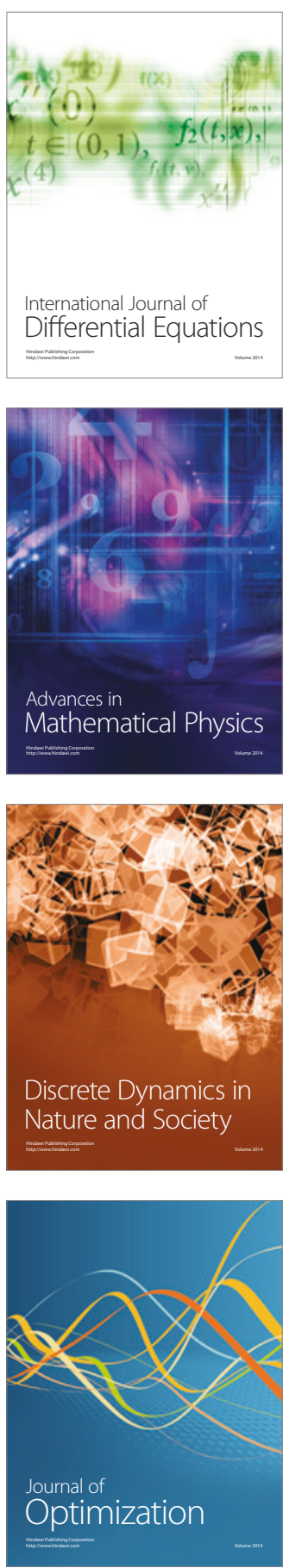